\section{Concomitant cat scratch disease and squamous cell carcinoma in a cardiac transplant}

\author{
Nitin Bhanot, ${ }^{1}$ George G. Sokos, ${ }^{2}$ \\ Raymond L. Benza, ${ }^{2}$ Srinivas Murali, \\ 1 Division of Infectious Diseases and \\ 2Division of Cardiology, Department of \\ Medicine, Allegheny General Hospital, \\ Pittsburgh
}

\section{Abstract}

Cat scratch disease has been reported very rarely in cardiac transplant recipients. In a review of 1073 episodes of infection in 620 heart transplant patients over a 16 year period, only one case of infection secondary to Bartonella henselae was documented. Another case of hepatosplenic bacillary angiomatosis from $B$. henselae was reported 2 decades ago in a heart transplant recipient who had presented with fevers of unknown origin. Although the typical clinical manifestation is that of a skin lesion accompanied with lymphadenopathy, cat scratch disease may present with persistent fevers without a clinically overt infective focus in immunosuppressed individuals. Moreover, more than one disease process may coexist in immunocompromised hosts. While the lymphadenopathy in our patient was secondary to Cat scratch disease, interestingly, the adjacent skin lesion that was thought to represent unhealed site of inoculation of Bartonella was diagnosed as squamous cell carcinoma.

\section{Introduction}

Cat scratch disease (CSD), caused by Bartonella henselae, has been reported very rarely in cardiac transplant recipients. We describe the case of a 65-year-old cardiac transplant recipient whose post transplant course was complicated with graft rejection, pulmonary nocardiosis, and post-transplantation lymphoproliferative disorder (PTLD). He presented with fevers and a skin lesion on his knee. A contrast enhanced CT scan of chest, abdomen, and pelvis was obtained that revealed lymphadenopathy in the left inguinal region. The suspicion for recurrence of PTLD was high, but consideration was given to CSD given the history of cat exposure and a skin lesion concomitant with lymphadenopathy, a common finding in CSD. The histopathologic testing, however, revealed lymphadenopathy secondary to CSD and the skin lesion due to squamous cell carcinoma.

\section{Case Report}

A 65-year-old man who had undergone orthotopic heart transplant for ischemic cardiomyopathy 2 years prior to presentation was admitted with fevers of 3-4 week duration. The fevers were mainly present in the evenings, ranging between $100^{\circ} \mathrm{F}$ and $102^{\circ} \mathrm{F}$, and were associated with chills and night sweats. The patient also complained of an intermittent dull aching headache in his occipital region. He reported constant fatigue that worsened with limited exertion. For the previous 6 months, the patient had noticed blurred vision, but denied any specific cardiac, respiratory, or abdominal complaints. There was no loss of weight or appetite. The rest of review of systems was unremarkable.

The patient's post cardiac transplant course was eventful. Three months after the transplant, he was diagnosed with pulmonary nocardiosis for which he completed 9 months of antimicrobial treatment. Two months later, he had cardiac graft rejection that was treated and remedied with methylprednisolone alone. A month post-rejection, he was diagnosed with post-transplant lymphoproliferative disorder and treated with rituximab. A PET-CT scan done 2 months prior to the current presentation did not reveal any evidence of malignancy. There was no history of sick contacts or recent travel. The patient had 2 dogs at home and a cat that had died 4 months earlier at the age of 14 years. His past medical history was significant for antiphospholipid antibody syndrome, hyperlipidemia, GERD, depression, hiatal hernia, and hypertension. His medication list included tacrolimus, aspirin, sirolimus, warfarin, citalopram, simvastatin, ezetimibe, omeprazole, calcium and vitamin D.

On examination, vital signs were within normal range except for an oral temperature of $101^{\circ} \mathrm{F}$. Fundoscopic exam performed by an ophthalmologist did not reveal any retinal lesions and there were no signs of meningismus. His chest exam revealed a well healed vertical scar from his transplant surgery. A non-tender $3 \mathrm{~cm}$ by $1.5 \mathrm{~cm}$ erythematous nodule with central scarring was seen over the left knee (Figure 1). The patient reported that the lesion was present for a couple of months, but was not sure about the exact time of onset of the lesion. Rest of the examination was essentially unremarkable.

Laboratory studies showed a normal CBC and comprehensive metabolic panel. His INR was 2.5. Blood and urine cultures did not reveal any microbial growth. A lumbar puncture was performed that was unremarkable. Given the most recent diagnosis of PTLD, the possibility of recurrence was entertained. A contrast enhanced CT scan of chest, abdomen, and pelvis was obtained that revealed lym-
Correspondence: Nitin Bhanot, Division of Infectious Diseases, Allegheny General Hospital, 320 East North Avenue, Pittsburgh, PA 15212, USA. Tel. +1.412.359.3683 - Fax: +1.412.359.6899 E-mail: nitinbhanot@gmail.com

Key words: Bartonella, cardiac, transplant.

Acknowledgements: the authors thank Dr. Katherine Jasnosz and Dr. Cunfeng Pu for help with the pathology slides; Dr. Ali Darabi with microbiologic assistance; and Ms. Kristin Petersen for help with the pictures.

Contributions: NB, GS, RL, and SM have contributed equally to writing of this manuscript. All the authors have read and approved the final manuscript. The case has not been submitted to and is not being considered for publication in any other journal.

Conflict of interest: the authors report no conflicts of interest.

Received for publication: 30 August 2011.

Revision received: 31 0ctober 2011.

Accepted for publication: 14 November 2011.

This work is licensed under a Creative Commons Attribution NonCommercial 3.0 License (CC BYNC 3.0).

(C) Copyright N. Bhanot et al., 2012

Licensee PAGEPress, Italy

Infectious Disease Reports 2012; 4:e2 doi:10.4081/idr.2012.e2

phadenopathy in the left inguinal region, with the largest node measuring $2 \mathrm{~cm}$ by $1.5 \mathrm{~cm}$. This heightened the suspicion for PTLD and an excisional biopsy of the lymph node was performed. Histopathology revealed necrotizing granulomas with paracortical plasmacytosis. Gram, GMS, and AFB stains were negative. Unfortunately, cultures on the pathologic specimen were not sent. Lymphocyte subtyping on the specimen did not suggest a diagnosis of lymphoma. Blood cultures for MAC, Quantiferon Gold, urine Histoplasma antigen, serum Cryptococcal antigen, and serologies for Histoplasma, Blastomyces, and Coccidioidomyces were sent. RPR and PCR on serum for EBV and CMV DNA were also ordered. All these tests yielded negative results. A transthoracic echocardiogram revealed normal valves with no evidence of intracardiac vegetations.

Given the histopathologic findings and history of previous contact with a cat, serology for Bartonella henselae was ordered. Concomitantly, dermatology was consulted for biopsy of the skin lesion. The serology for Bartonella henselae returned positive for both IgM (1:200; negative: $<100)$ and $\operatorname{IgG}(>1: 2,560$; negative $:<$ 1:320). The skin lesion on the knee proximal lymphadenopathy was presumed to represent 
an unhealed site of inoculation of the bacterium. In contradistinction to our initial diagnostic impression, the histopathologic findings on the skin biopsy revealed infiltrating strands of atypical squamous epithelium within the dermis, highly suggestive of squamous cell carcinoma (Figure 2). A wide excision of the lesion was subsequently performed that confirmed the diagnosis of a skin malignancy. Fortunately, the margins of the lesion were free of tumor. We retrospectively requested a Warthin-Starry stain on the nodal specimen which revealed coccobacillary pathogens present singly and in clumps, suggestive of Bartonella spp (Figure 3).

The patient was initiated on doxycycline and azithromycin as soon as the serology turned positive, and he responded remarkably to the antimicrobial therapy. Twenty four hours after initiation of the antibiotics, his fevers abated

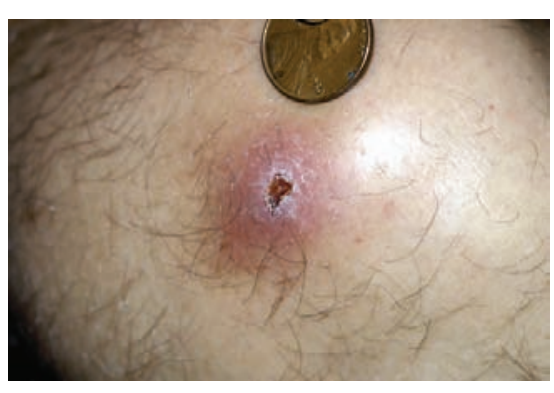

Figure 1. An erythematous nodule with central scabbing over the left knee.

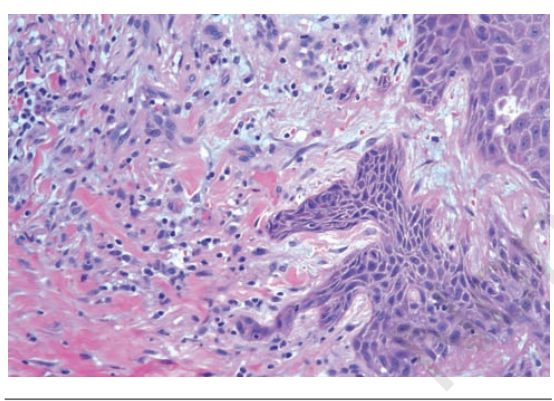

Figure 2. Histopathology of the skin lesion revealing infiltrating atypical squamous cells into the deeper stromal tissue (H\&E stain).

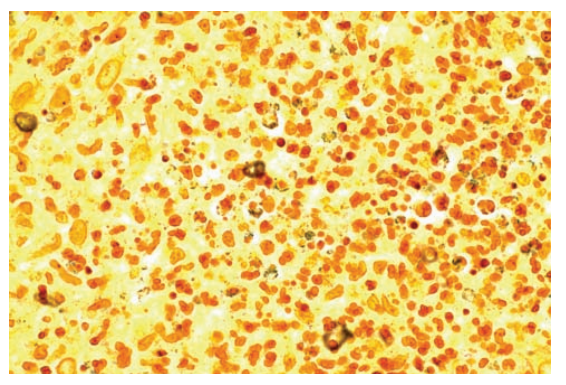

Figure 3. Short curved microorganisms seen singly and in clusters on the lymph node specimen (Warthin-Starry stain). and his fatigue, night sweats and headache resolved within a few days. Doxycycline was stopped after 3 weeks and azithromycin was continued for a total of 3 months.

\section{Discussion}

CSD in heart transplant recipients is a very rare entity. ${ }^{1}$ Most cases have been reported in patients who have had renal transplants. ${ }^{1}$ Cases have been described in liver and lung transplant recipients as well. ${ }^{2,3}$ Kemper et al. described a case of hepatosplenic bacillary angiomatosis from $B$. henselae 2 decades ago in a cardiac transplant recipient who had presented with a fever of unknown origin. ${ }^{4}$ Montoya et al. analyzed a total of 1073 episodes of infection in 620 heart transplant patients over a 16 year period and found only one case of infection secondary to Bartonella henselae (details of the case were not published in the article). ${ }^{5}$ To the best of our knowledge, no other cases of CSD in a heart transplant recipient have been reported in the medical literature published in English language to date.

Other than the rarity of CSD in cardiac transplant recipients, what makes the case interesting is the presence of a malignancy concomitant with an infection. Bartonella can cause a variety of skin lesions and an erythematous papule or nodule with crusting or ulceration is commonly seen with CSD. ${ }^{6}$ Lymphadenopathy is often seen in the lymph nodes that drain the area of the skin affected, a clinical picture seen in the current case. ${ }^{7}$ Before the results of skin biopsy were obtained, we presumed that the skin lesion probably represented an unhealed site of bacterial inoculation. The coexistence of malignancy and infection in this patient was probably secondary to immunosuppression in this cardiac transplant recipient.

Infections from Bartonella may present atypically and mimic a malignancy clinically as well as radiologically. ${ }^{8,9}$ They may masquerade as a lymphoma and can be mistaken for PTLD in organ transplant recipients. ${ }^{8,10}$ This was a consideration in our patient because he had a prior history of PTLD. Rarely, even the histopathologic findings associated with lesions from Bartonella may be mistaken for a malignancy. Pseudoepitheliomatous hyperplasia, a term described to denote a marked reactive hyperplasia of the epidermis, may mimic squamous cell carcinoma. A rare association between pseudoepitheliomatous hyperplasia and Bartonella infection has been reported in the medical literature. ${ }^{11}$ This diagnosis was considered in the differential, but the pattern of infiltration by atypical squamous cells into the deeper stromal tissue confirmed that the underlying disease process was a malignancy and not reactive hyperplasia alone. Finally, it has also been demonstrated that Bartonella spp stimulate cellular proliferation and may lead to tumor formation. ${ }^{12,13}$ We requested a Warthin-Starry stain and PCR for Bartonella on the skin lesion. Both the tests were negative, however. PCR for Bartonella on the nodal specimen was not performed as there was no available tissue. PCR on the skin lesion was performed 2 weeks after the initiation of the antibiotics and may have affected the result.

In addition to the association of Bartonella with malignancy, this bacterium has also been associated with rejection in transplant recipients. Dharnidharka et al. reported a possible link between acute rejection and CSD in 2 patients after pediatric renal transplantation. ${ }^{10}$ No similar association, however, has been reported since.

While in otherwise healthy individuals CSD presents as a skin lesion and lymphadenopathy that may resolve spontaneously, in the immunosuppressed hosts, the disease may progress and present atypically. It may present as FUO and involve visceral organs such as liver, spleen, bone, heart, and lungs..$^{7,14-15}$ Therefore, it is important to obtain a detailed history in patients who present with FU0. Bartonella rarely grows on routine bacterial cultures and if tests such as special stains (Warthin-Starry or Steiner or Dieterle stain), serology for Bartonella, or PCR on tissue and/or blood are not sent, diagnosis may remain elusive. ${ }^{7}$ The typical history of scratch from a cat may not be present and absence of this should not preclude one from entertaining a diagnosis of Bartonellosis. Close contact with cats alone, along with fever and lymphadenopathy should raise a suspicion for it. ${ }^{7}$ Epidemiological, clinical, and histological data should be obtained and definitive diagnostic testing such as special stains, serology and/or PCR should be ordered in the right setting. ${ }^{7,15}$ Our patient did not recall that his cat had bitten or scratched him ever. He did admit that the animal frequently slept with him in his bed.

While immunocompetent individuals may not even need treatment for CSD, it is generally recommended that those with immunodeficiency states such as AIDS and transplantation received prolonged courses (at least 3 months) of antimicrobial therapy. ${ }^{16}$ Short courses of therapy that may suffice in otherwise healthy hosts may not be adequate in transplant recipients. Rheault et al. treated a renal transplant patient with a 5 day course of azithromycin and witnessed a relapse after 8 months. ${ }^{17}$

In addition to the rarity of CSD in cardiac transplant recipients, certain aspects of the case are worth noting. Firstly, there was an unusually long latent period between the introduction of infection and the clinical presentation, the cause of which remains elusive. 
Secondly, the case demonstrates the possibility of coexistence of malignancy and infection in immunosuppressed hosts. The differential diagnosis of fever in immunosuppressed individuals is wide and more than one disease process may coexist in these hosts. Finally, the case highlights the importance of history and physical examination in diagnosing patients who present with prolonged fevers. Despite the advancement of newer diagnostic techniques, a detailed history and physical examination is of paramount importance in clinical diagnosis. Radiological and invasive testing should supplement clinical judgment to make definitive diagnoses.

\section{References}

1. Kotton CN. Zoonoses in solid-organ and hematopoietic stem cell transplant recipients. Clin Infect Dis 2007;44:857-66.

2. Thudi KR, Kreikemeier JT, Phillips NJ, et al. Cat scratch disease causing hepatic masses after liver transplant. Liver Int 2007;27:145-8.

3. Lienhardt B, Irani S, Gaspert A, et al. Disseminated infection with Bartonella henselae in a lung transplant recipient. $\mathrm{J}$ Heart Lung Transplant 2009;28:736-9.

4. Kemper CA, Lombard CM, Deresinski SC,
Tompkins LS. Visceral bacillary epithelioid angiomatosis: possible manifestations of disseminated cat scratch disease in the immunocompromised host: a report of two cases. Am J Med 1990; 89:216-22.

5. Montoya JG, Giraldo LF, Efron B, et al. Infectious complications among 620 consecutive heart transplant patients at Stanford University Medical Center. Clin Infect Dis 2001;33:629-40.

6. Chian CA, Arrese JE, Piérard GE. Skin manifestations of Bartonella infections. Int J Dermatol 2002;41:461-6.

7. Slater LN, Welch DF. Bartonella, Including Cat-Scratch Disease. In: Mandell GL, Bennett JE, Dolin R, editors. Principles and Practice of Infectious Diseases. 7th edition. Philadelphia: Elsevier; 2010. pp 2995-3009.

8. Ghez D, Bernard L, Bayou E, et al. Bartonella henselae infection mimicking a splenic lymphoma. Scand J Infect Dis 2001;33:935-6.

9. Kempf VA, Petzold H, Autenrieth IB. Cat scratch disease due to Bartonella henselae infection mimicking parotid malignancy. Eur J Clin Microbiol Infect Dis 2001;20: 732-3.

10. Dharnidharka VR, Richard GA, Neiberger RE, Fennell RS 3rd. Cat scratch disease and acute rejection after pediatric renal transplantation. Pediatr Transplant 2002;6: 327-31.
11. Amsbaugh S, Huiras E, Wang NS, et al. Bacillary angiomatosis associated with pseudoepitheliomatous hyperplasia. Am J Dermatopathol 2006;28:32-5.

12. Dehio C. Bartonella-host-cell interactions and vascular tumour formation. Nat Rev Microbiol 2005;3:621-31.

13. Vassallo $\mathrm{C}$, Ardigò $\mathrm{M}$, Brazzelli $\mathrm{V}$, et al. Bartonella-related pseudomembranous angiomatous papillomatosis of the oral cavity associated with allogeneic bone marrow transplantation and oral graft-versus-host disease. Br J Dermatol 2007;157: 174-8.

14. Lamps LW, Scott MA. Cat-scratch disease: historic, clinical, and pathologic perspectives. Am J Clin Pathol 2004;121Suppl:S7180.

15. Hansmann Y, DeMartino S, Piémont Y, et al. Diagnosis of cat scratch disease with detection of Bartonella henselae by PCR: a study of patients with lymph node enlargement. J Clin Microbiol 2005;43:3800-6.

16. Koehler JE, Duncan LM. Case records of the Massachusetts General Hospital. Case 30-2005. A 56-year-old man with fever and axillary lymphadenopathy. N Engl J Med 2005;353:1387-94.

17. Rheault MN, van Burik JA, Mauer M, et al. Cat-scratch disease relapse in a kidney transplant recipient. Pediatr Transplant 2007;11:105-9. 